\title{
MOVIMENTAÇÃO E DIFERENCIAÇÃO NARRATIVA AO LONGO DO PROCESSO TERAPÊUTICO: UM ESTUDO DE CASO LONGITUDINAL À LUZ DO MODELO DE COMPLE- MENTARIDADE PARADIGMÁTICA
}

\author{
Joana Fojo Ferreira ${ }^{2}$ \\ Nuno Conceição ${ }^{1}$ \\ António Branco Vasco ${ }^{1}$
}

\begin{abstract}
Resumo: As narrativas são frequentemente consideradas o meio por excelência de reconhecimento e reorganização da experiência interna dos pacientes.

Neste estudo procurou-se diferenciar e identificar potenciais marcadores narrativos de capacidade de processamento e, ao quantificá-los em diferentes momentos do processo terapêutico, verificar se existia evidência a favor da proposta de sequencialização do trabalho terapêutico do Meta-modelo de Complementaridade Paradigmática, em termos de estratégias gerais.

Foram definidos, reconhecidos e analisados processos narrativos a nível molecular (a partir da revisão de literatura) e a nível molar (a partir da teoria do meta-modelo supra-referido). Em ambos os casos, enfatizou-se as formas e capacidades de processamento da informação em detrimento dos conteúdos narrados.

Os resultados evidenciaram diferenciação e movimentação entre as fases consideradas para processos moleculares e molares, e nestes últimos reconheceu-se um padrão de progressão narrativa de acordo com a sequencialidade proposta pelo modelo, para as fases em estudo.
\end{abstract}

Palavras-chave: Sequencialização; Fases do Processo; Processos Narrativos; Marcadores.

Narrative Progress and Differentiation along the Therapeutic Process: A Longitudinal Case-Study within the Paradigmatic Complementarity Model (Abstract): Narratives are frequently considered the best means for recognizing and reorganizing patients' internal experience.

In this study we tried to differentiate and identify potential narrative markers of processing capacity and, by quantifying them at different moments in the thera-

\footnotetext{
${ }^{1}$ Universidade de Lisboa

2 joanafojo.ferreira@gmail.com

PSICOLOGIA, Vol. XXV (1), 2011, Edições Colibri, Lisboa, pp. 173-201
} 
peutic process, check if there was evidence in favor of the proposal of sequencing of the therapeutic work, in terms of general strategies, as postulated by the Meta-model of Paradigmatic Complementarity.

Narrative processes were defined, recognized and analyzed at a molecular level (based on literature review on narrative) and at a molar level (based on the meta-model identified above). In both cases processing capacities were emphasized rather than the narrated contents.

Both at the molecular and molar levels, the results show differentiation and movement along the phases under scrutiny. Besides, a pattern of narrative progression was recognized at the molar level, according to the sequence proposed by the meta-model regarding the phases tapped.

Key words: Sequencing; Process Phases; Narrative Processes; Markers.

\section{Movimentação e Diferenciação Narrativa ao Longo do Processo Terapêutico: Um Estudo de Caso Longitudinal à Luz do Modelo de Complementaridade Paradigmática}

O movimento integrativo em psicoterapia tem permitido visões progressivamente mais abrangentes dos pacientes e dos problemas e reflecte o reconhecimento da importância de adequar as intervenções aos pacientes mais do que encaixá-los nas abordagens interventivas preferenciais (Beutler, Consoli, \& Lane, 2005; Gold, 1994; Lazarus, 2005; Moleiro, 2005; Norcross, 2005; Vasco, 2001, 2006). Intensificou, assim, o foco nas características dos pacientes (Beutler et al., 2005; Lambert \& Bergin, 1994) e introduziu a necessidade de avaliações ao longo do processo terapêutico, reconhecendo que estas características não são necessariamente estáticas e que as necessidades de trabalho podem ser diferentes em momentos diferentes da terapia (Gold, 1994; Lazarus, 2005; Prochaska \& DiClemente, 2005; Vasco, 2001, 2006). Esta perspectiva remete-nos para a importância de considerar as modificações e o movimento das necessidades dos pacientes e dos objectivos e trabalho terapêuticos ao longo do processo terapêutico.

O reconhecimento do processo terapêutico como um processo evolutivo tem levado vários autores a investigar e defender a importância de, não só avaliar as necessidades dos pacientes, mas também os ganhos terapêuticos já conquistados, possibilitadores de conquistas posteriores (Conceição, 2005; Stiles et al., 1991; Tallman \& Bohart, 1999; Toukmanian, 1986). Este é, também, um dos pressupostos do Meta-modelo de Complementaridade Paradigmática (MCP) onde se insere o presente estudo (Vasco, 2001, 2006). 


\section{Meta-modelo de Complementaridade Paradigmática}

O MCP é um modelo meta-integrativo, que contempla quatro componentes:

“(a) "princípios gerais de mudança terapêutica" (o "quê" da terapia); (b) a aliança terapêutica (o "estar" em terapia); (c) "conceptualização do paciente e do problema" (o "quê" mais o "como" da terapia); e (d) "sequência temporal de fases relativas a objectivos" (o "quando" da terapia)." (Vasco, 2006, p. 15).

O presente estudo assenta primordialmente neste último componente, não desconsiderando, ainda assim, as características do paciente, o problema apresentado e a relação e trabalho terapêuticos.

$\mathrm{O}$ modelo postula sete fases tendencialmente sequenciais de mudança terapêutica. Crê-se que numa sessão um terapeuta possa promover estratégias gerais ou objectivos estratégicos de várias dimensões, mas que exista uma predominância num determinado tipo de estratégias gerais, estando a ideia de fase associada a essa predominância.

As fases do modelo são: 1 - Confiança, motivação e estruturação da relação; 2 - Aumento da consciência da experiência e do Self; 3 - Construção de novos significados relativos à experiência e ao Self; 4 - Regulação da responsabilidade; 5 - Implementação de acções reparadoras; 6 - Consolidação da mudança; e 7 - Antecipação do futuro e prevenção da recaída. Cabe ao terapeuta determinar quando promover predominantemente que objectivos estratégicos específicos de que fase.

Conceição e Vasco (2008) esquematizaram a versão actual dos objectivos estratégicos (baseados em factores comuns) para a sequencialização do processo terapêutico segundo uma pirâmide de sete fases em sentido crescente (Apêndice A). Estes objectivos estratégicos (ou estratégias gerais) podem ser vistos como trabalho terapêutico a promover (ou a não promover) e no sentido da capacitação de competências nos pacientes e de diminuições de vulnerabilidades.

Os estudos empíricos na componente "fases" do MCP dos últimos 10 anos têm sustentado esta dimensão temporal relativa aos objectivos estratégicos (Conceição, 2005; Conceição \& Vasco, 2009; Vasco, 2006). Têm também dado conta das potencialidades da prossecução sequencial dos objectivos estratégicos na intervenção com perturbações sérias da personalidade (Gonçalves \& Vasco, 2001), apontado para um aumento da estimativa de melhoras por parte do paciente à medida que este avança nas fases do modelo (Vasco, 2006), e têm alertado para a importância de articular a promoção (por parte do terapeuta) com a capacidade (por parte do paciente) para 
melhores alianças e resultados terapêuticos (Conceição, 2005; Conceição \& Vasco, 2009).

Uma vez que o modelo defende que, por mais que o paciente necessite de desenvolver competências de fases mais avançadas, os resultados terapêuticos são mais sólidos quando as competências de fases precedentes estão suficientemente asseguradas (Osatuke et al., 2004), torna-se importante avaliar nos pacientes a presença de indicadores, ou marcadores, de capacidades de processamento já adquiridas. Crê-se que esses marcadores poderão apoiar uma tomada de decisão mais fundamentada, relativamente à pertinência de avançar para trabalho de promoção de objectivos de fases posteriores, ou de consolidação dos de fases anteriores. A questão central de investigação do presente artigo prende-se com se porventura esses marcadores de capacidade de processamento estarão evidentes nas narrativas dos pacientes.

\section{Capacidade de Processamento}

Assemelhamos capacidade de processamento ao processo Piagetiano de acomodação (e.g. Battro, 1978; Lourenço, 2005; Piaget, 1977), uma vez que procuramos reconhecer indicadores de mudança nas formas habituais de processamento, já bem integradas no reportório dos pacientes, e não apenas anexação do que é promovido pelo terapeuta nos esquemas de significação habituais (assimilação). Contudo, este estudo assume uma postura mais neo-piagetiana, que congrega a teoria Piagetiana com os modelos de processamento da informação, acreditando que esta capacidade/acomodação é reflectida em mudanças nos processos de atenção e memória e nas estratégias de tratamento da informação, e pode ser de natureza cognitiva, emocional, sensorial e/ou interpessoal (Greenberg \& Pascual-Leone, 2001).

\section{Processos narrativos}

No sentido de estudar a evolução na capacidade de processamento dos pacientes ao longo do processo terapêutico, julgámos pertinente analisar os processos narrativos presentes em momentos diferentes da terapia, uma vez que as mudanças terapêuticas são acompanhadas de mudanças narrativas, e a progressiva riqueza, profundidade e diferenciação das narrativas permite avaliar e compreender a mudança no paciente (Hardtke \& Angus, 2004; Osatuke et al., 2004).

Esta ênfase nos processos narrativos advém da nossa identificação com Piaget (citado em Lourenço, 2005), que dá prioridade ao "conhecimento estrutural" (o modo de conhecer e pensar) em detrimento do conhecimento factual (os conhecimentos adquiridos em si).

Também Greenberg, Rice e Elliott (1993) apresentam o conceito diagnóstico de processo, defendendo que não é a modificação ou compreen- 
são de conteúdos específicos que prediz bons resultados terapêuticos, mas antes a análise e intervenção no modo corrente de processamento cognitivo-afectivo do paciente. Postulam que as decisões dos terapeutas se devem basear no como os clientes organizam a sua experiência no momento, e que o trabalho interventivo deve apostar na facilitação da mudança na forma de processamento dos indivíduos, modificando aquilo a que estão atentos e como o simbolizam. A título de exemplo, sabendo como promover um processamento no paciente que repare ou transforme as suas clivagens de auto-crítica em instâncias de auto-aceitação, é possível facilitar-se esse mesmo processo em cinquenta pacientes, independentemente dos conteúdos específicos das auto-críticas serem diferentes em cada um. Isto é, em vez de se trabalhar ao nível do conteúdo da auto-crítica, trabalha-se ao nível do processo da auto-crítica, ainda que para isso se atenda aos conteúdos, naturalmente.

\section{Modelos de Evolução do Processo Terapêutico}

Qualquer processo evolutivo, de desenvolvimento ou de mudança, pode dar-se em diferentes escalas temporais, desde segundos, minutos, horas, semanas, meses ou anos. O presente estudo situa-se na metade direita do contínuo: semanas, meses e anos; no entanto, diversos modelos, mesmo situados na metade esquerda do contínuo, contribuíram para a projecção deste estudo.

Destacamos o Modelo de Assimilação (Detert, Llewelyn, Hardy, Barkham, \& Stiles, 2006; Honos-Webb, Stiles, \& Greenberg, 2003; Osatuke et al., 2004; Stiles et al., 1990, 1991; Stiles, Honos-Webb, \& Lani, 1999) que se assemelha ao MCP no que concerne a especificação de passos sequenciais, embora se constrinja à exploração e resolução de áreas problemáticas específicas, identificadas momento-a-momento. Sustenta oito estádios sequenciais de assimilação de experiências problemáticas, com narrativas distintas para cada estádio: Estádio 0 - Evitante/dissociado, Estádio 1 - Pensamentos indesejados/ evitamento activo, Estádio 2 - Consciência vaga/ emergência, Estádio 3 - Afirmação do problema/clarificação, Estádio 4 Compreensão/insight, Estádio 5 - Aplicação/lidar com, Estádio 6 - Apropriação de recursos/resolução do problema, Estádio 7 - Integração/mestria.

Atrevemo-nos a apontar que o modelo de Assimilação procura promover, para histórias/experiências particulares, o que o MCP procura capacitar de forma mais geral. Neste sentido, é uma evolução narrativa semelhante à do Modelo de Assimilação que esperamos encontrar nas sessões terapêuticas segundo o $\mathrm{MCP}$, com a diferença de não considerarmos manifestações esporádicas como reflexo de capacidade, mas procurando, outro sim, manifestações regulares e transversais a diferentes situações apresentadas. 
Neste sentido, o estudo de Rudolf et al. (2002), que sustenta uma escala de sete fases, Heidelberg Structural Change Scale (HSCS), equivalente ao Modelo de Assimilação mas adaptado à abordagem psicanalítica, apresenta um estudo de caso, no qual, paralelamente ao estudo momento-a-momento da evolução de um problema-alvo, é possível comparar a evolução temporal para problemas-alvo diferentes. Neste estudo, verifica-se, por um lado, uma evolução sequencial para todos os problemas e, por outro, uma proximidade temporal em termos do posicionamento na HSCS para os diferentes problemas. Esta última verificação já permite extrapolar para um nível mais molar, assente em mudanças fase-a-fase e menos momento-a-momento, sugerindo que, num processo terapêutico (pelo menos psicanalítico) em que surgem diversos problemas, os pacientes tendem a evoluir paralelamente nas diversas áreas e não a resolver um problema de cada vez. Como é sugerido pelo $\mathrm{MCP}$, parece fazer sentido capacitar os pacientes para um determinado nível de processamento nas várias áreas problemáticas, mais do que voltar ao nível inicial para cada novo problema.

Também o Modelo de Processo Narrativo (Angus, Levitt, \& Hardtke, 1999; Angus, Lewin, Bouffard, \& Rotondi-Trevisan, 2004) nos possibilitou reflexões interessantes. Este modelo defende que a mudança terapêutica implica movimentos dialécticos entre três processos narrativos - a descrição de eventos (sequências narrativas externas), a diferenciação emocional (sequências narrativas internas) e a reflexão e articulação daqueles para a atribuição de significado (sequências narrativas reflexivas). Diferentes abordagens, todas com ganhos terapêuticos, sequenciam diferentemente os processos a promover (Angus et al., 1999).

$\mathrm{O}$ trabalho destes autores parece desafiar a tendência à sequencialidade postulada pelo MCP, contudo, as possibilidades de comparação dos dois modelos poderão ser limitadas, o Modelo de Processo Narrativo parece ser mais transversal aos vários momentos da terapia, sendo possível promover estes movimentos dialécticos em cada uma das fases do Meta-modelo.

Ainda no âmbito do trabalho de Angus e colaboradores, destacamos Angus, Bryntwick, Boritz, Warwar, \& Greenberg (2008), em que discriminam marcadores narrativos de emoção, metáforas em que enquadram tipos específicos de narrativas, como Untold Story ou Unique Outcome Story. O reconhecimento e nomeação de marcadores narrativos, que funcionam como categorias onde se podem incluir diferentes conteúdos, aproxima-se do pretendido com este estudo.

Outros estudos na área trazem contributos pertinentes. Um deles refere-se ao Modelo Psicoterapêutico Cognitivo-Narrativo (Gonçalves, Henriques, \& Machado, 2004; Moreira, Beutler, \& Gonçalves, 2008), que sugere três fases de trabalho sequenciais: recolha, adjectivação e projecção. Outro estudo refere-se aos Níveis de Processamento Perceptual dos Clientes 
(Toukmanian, 1986), que apontam para sete níveis sequenciais de processamento dos pacientes ao longo da terapia, desde afirmações indiferenciadas e externalizadas, a afirmações mais diferenciadas, internalizadas e integradas. Baptista (2000), por sua vez, elabora sobre o tipo de narrativas presentes em cada estádio de Mudança de Prochaska: Pré-Contemplação - narrativas impermeáveis à mudança, dada a falta de consciência quanto às suas consequências; Contemplação - narrativas exteriorizadas; Preparação - o indivíduo demonstra estar preparado para mudar parte da narrativa; Acção mudança activa das narrativas, com experimentação de narrativas alternativas (adopção de diferentes papéis, antecipação de selves possíveis, consideração de diferentes projecções no futuro); e Manutenção - narrativas mais estáveis e adequadas à pessoa.

\section{Processos moleculares e molares}

Ao longo do estudo, fomos tomando como que duas frentes de trabalho, uma assente na investigação no que consideramos serem processos narrativos mais moleculares e outra assente no que consideramos serem processos narrativos mais molares. Para clarificar, estabelecemos a analogia com os conceitos biológicos de célula e de tecido, respectivamente: as células integram os tecidos e são fundamentais para que os tecidos exerçam as suas funções, mas é difícil distinguir dois tecidos analisando uma célula de cada um, que sem o contexto do tecido poderiam parecer-nos iguais. Movimentos dialécticos entre estes dois níveis de análise são importantes: ir ao molecular para perceber como é que o molar é constituído, e voltar ao molar para perceber a arquitectura geral.

No presente estudo, os processos moleculares estão associados aos processos mais transversais, identificados momento-a-momento, como por exemplo o processo de diferenciação emocional (Angus et al., 2004) e o processo de subjectivação cognitiva (Gonçalves et al., 2004), e os processos molares estão associados aos objectivos estratégicos das fases do Meta-modelo, mais abrangentes mas também mais diferenciáveis ao longo do processo.

O objectivo do estudo prende-se com a identificação e diferenciação de marcadores narrativos de capacidade de processamento, verificada ao longo de um processo terapêutico com base no MCP, de forma a avaliar se existe evolução dos processos narrativos ao longo do processo terapêutico de acordo com a proposta de sequencialização de fases do referido meta-modelo. De certa forma, procuramos verificar se os objectivos estratégicos de cada fase do MCP são traduzíveis em processos narrativos, ou indicadores verbais do paciente, que funcionem como marcadores de conquistas tera- 
pêuticas intermédias, reflexo de capacidade de processamento, e se existe uma evolução nesses processos narrativos ao longo do processo terapêutico de acordo com a sequência de fases proposta. Sendo um meta-modelo que tem tido algum apoio empírico desde 1999, os resultados deste estudo poderão, paralelamente, corroborar, descartar ou aprimorar a identidade/definição de cada fase e a sequência terapêutica proposta.

\section{Método}

Procedeu-se à análise longitudinal de um estudo de caso no sentido de investigar as variações intra-individuais ao nível do processamento narrativo. Não se trata, contudo, de um estudo de caso nos moldes tradicionais de avaliação do trabalho e eficácia terapêuticos; trata-se, isso sim, de um estudo de caso ao serviço da corroboração (ou desconfirmação) da sequencialidade proposta pelo $\mathrm{MCP}$. O foco é, por isso, no processamento do paciente em momentos diferentes do processo terapêutico, independentemente da eficácia da terapia em si, ainda que a eficácia possa ser inferida com base na evolução narrativa.

A paciente consentiu, por escrito, a realização do estudo, incluindo gravação e cotação das sessões, mediante certificação da confidencialidade no que concerne dados identificativos. Foram, nomeadamente, partilhados com ela os excertos do seu discurso que apresentamos no artigo de forma a assegurar que ela não se sentia demasiado exposta.

\section{Paciente}

A paciente é uma adulta do sexo feminino, com 29 anos à data do início do processo, licenciada e com uma ocupação profissional na área da saúde mental.

Procurou ajuda por razões de desenvolvimento pessoal, sem queixas sintomáticas marcadas, apresentando traços evitantes e dependentes de personalidade. Apresentava queixas de ordem depressiva e de insatisfação conjugal na sua relação com o parceiro com quem namorava há nove anos, e dificuldade de diferenciação, de exposição e de afirmação face aos outros.

No caso desta paciente, o terapeuta optou deliberadamente por não utilizar quaisquer instrumentos de avaliação formais.

À data da conclusão deste estudo, o processo ainda decorria e tinha já a duração de 3 anos, com sessões semanais. 


\section{Terapeuta}

O terapeuta é o segundo autor deste estudo, trabalha em clínica privada, e tinha 33 anos e 10 anos de experiência clínica à data da última sessão observada. Tem formação em várias abordagens psicoterapêuticas, considera-se um terapeuta integrativo que procura respeitar os princípios de tomada de decisão clínica postulados pelo MCP, e integra princípios e estratégias de abordagens experienciais e existenciais, motivacionais e relacionais, e cognitivo-comportamentais.

\section{Procedimento}

Inicialmente, categorizando-se os processos reconhecidos na literatura, identificaram-se os processos narrativos a verificar nas sessões terapêuticas, tendo-se chegado aos processos enunciados na Figura 1.

Estes processos foram considerados na construção do protocolo de análise (Apêndice B), a ser preenchido pelo avaliador imediatamente após a visualização da sessão, onde se classificam, numa escala de 0 a 8 , os processos narrativos reconhecidos em cada sessão terapêutica visualizada.

Seguidamente, procedeu-se à selecção do caso. De uma base de sessões gravadas em vídeo utilizadas em Conceição (2010), seleccionou-se, aleatoriamente, um dos casos cotados nas fases do MCP por avaliadores independentes do estudo actual. Nenhum dos casos disponíveis inicialmente tinha gravações desde o início do processo ou estava finalizado, facto que não interfere com os objectivos do presente estudo.

Para a selecção das sessões a visualizar, esquematizaram-se graficamente as várias sessões, sobrepondo a promoção do terapeuta com a capacidade do paciente. Discriminaram-se as sessões que combinavam um grau médio-elevado de promoção do terapeuta numa determinada fase, com um grau médio-elevado de capacidade da paciente na mesma fase. A intenção foi garantir que, não só a paciente já tinha alcançado os ganhos terapêuticos específicos de determinada fase, como, através da promoção do terapeuta, estes eram trabalhados (surgiam) em sessão. Visualizaram-se e analisaram-se 9 sessões terapêuticas, em momentos diferentes do processo terapêutico: três sessões predominantemente em fase 2 , três predominantemente em fase $3 \mathrm{e}$ outras três predominantemente em fase 4 . A fase predominante das sessões foi calculada em Conceição (2010) com base nas avaliações de três juízes que utilizaram o Inventário de Estratégias Gerais na perspectiva de observador (IEG-O, Conceição \& Vasco, 2008), que faz parte de uma bateria de instrumentos de avaliação associada ao MCP. Entre outras dimensões não relevantes para este estudo, O IEG-O é composto por 2 conjuntos de sete 
Figura 1: Descrição dos Processos Narrativos considerados na elaboração do protocolo de análise das sessões terapêuticas a visualizar

\begin{tabular}{|c|}
\hline Abstracção/Generalização \\
\hline $\begin{array}{l}\text { Aplicação a diversos contextos. } \\
\text { Relatos abstractos, sem concretização. }\end{array}$ \\
\hline Afirmação \\
\hline $\begin{array}{l}\text { Reconhecimento firme e convicto da sua individualidade, dos seus direitos e/ou } \\
\text { defesa activa destes. }\end{array}$ \\
\hline Atribuição de significado \\
\hline $\begin{array}{l}\text { Desenvolvimento de uma metáfora, de um sentido para sentimentos, pensamentos, } \\
\text { comportamentos, acontecimentos, por si mesmos ou em articulação uns com os } \\
\text { outros. }\end{array}$ \\
\hline Categorização \\
\hline $\begin{array}{l}\text { Agrupamento de ideias, pensamentos, sentimentos, comportamentos, acontecimen- } \\
\text { tos segundo características comuns. } \\
\text { Classificaça de pensamentos, sentimentos, comportamentos elou acontecimentos. }\end{array}$ \\
\hline Consciencialização \\
\hline $\begin{array}{l}\text { Apropriação de algo novo, seja um sentimento, pensamento, comportamento, situa- } \\
\text { ção, ou um conjunto de sentimentos, pensamentos, comportamentos elou situações. }\end{array}$ \\
\hline Contextualização \\
\hline $\begin{array}{l}\text { Enquadramento dos sentimentos, pensamentos, comportamentos e acontecimentos } \\
\text { nos seus devidos contextos/circunstâncias. }\end{array}$ \\
\hline Descrição \\
\hline $\begin{array}{l}\text { Relato de acontecimentos, sentimentos, pensamentos, comportamentos, com alguma } \\
\text { ordem. }\end{array}$ \\
\hline Diferenciação \\
\hline $\begin{array}{l}\text { Estabelecimento de diferenças entre sentimentos, pensamentos, acontecimentos } \\
\text { elou comportamentos, enquadrando-os nos seus momentos. }\end{array}$ \\
\hline Elaboração \\
\hline $\begin{array}{l}\text { Reflexão sobre uma ideia (sentimentos, pensamentos, comportamentos, aconteci- } \\
\text { mentos) ou um conjunto de ideias. Análise das ideias. }\end{array}$ \\
\hline Especificação/concretização \\
\hline $\begin{array}{l}\text { Pormenorização ou materialização de sentimentos, pensamentos, comportamentos, } \\
\text { acontecimentos. Exemplificação de situações. }\end{array}$ \\
\hline Estabelecimento de relações \\
\hline $\begin{array}{l}\text { Integração de ideias, pensamentos, sentimentos, comportamentos elou aconteci- } \\
\text { mentos; estabelecimento de conexões. }\end{array}$ \\
\hline Exploração \\
\hline Investigação, procura activa de esclarecimento. \\
\hline Responsabilização \\
\hline $\begin{array}{l}\text { Reconhecimento do seu papel/contributo nos acontecimentos da sua vida, nos seus } \\
\text { sentimentos, pensamentos e comportamentos. }\end{array}$ \\
\hline
\end{tabular}


escalas tipo Likert de 10 pontos, correspondentes às sete fases do Meta-modelo. O primeiro conjunto corresponde às operações do terapeuta, demonstradas na sessão, em termos dos objectivos estratégicos que está a promover, e o segundo conjunto corresponde à capacidade demonstrada pelo paciente nos mesmos objectivos estratégicos. A escala que obtém o valor mais alto ao nível das operações do terapeuta é dada como a fase predominante (Conceição, 2005). O treino e o procedimento de cotação dos juízes associados a esta avaliação é descrito em Conceição (2010) e o índice de acordo entre os juízes $\operatorname{ICC}(3,3)$ (Shrout \& Fleiss, 1979) é superior a .90 nas avaliações do caso utilizado no presente estudo.

Nenhuma das 9 sessões segue imediatamente a anterior, existindo, pelo menos, 5 semanas entre cada uma (foram visualizadas as sessões 31,36 , $46,51,65,80,90,100$ e 105). Cronologicamente, as sessões em fase predominante 2 precedem as sessões em fase predominante 3 , que, por sua vez, precedem as sessões em fase predominante 4. Não foram visualizadas sessões em fase 1, dada a inexistência de registos de vídeo destas sessões (só se havia negociado permissão para gravar o processo volvidos já seis meses de terapia, sensivelmente 24 sessões), nem de fase 5 e posteriores, uma vez que a paciente se encontrava em fase predominante 4. Além do mais, enquanto as 6 últimas sessões são claramente representativas das fases 3 e 4, as primeiras três sessões não são tão específicas da fase 2 , estando já a paciente em transição para a fase 3. Mais uma vez, por falta de material audiovisual, não foi possível seleccionar sessões francamente representativas da fase 2 . Também, apesar das últimas três sessões representarem, claramente, a fase 4 , a paciente ainda se encontrava nesta fase do processo, pelo que a sua capacidade nesta fase é ainda reduzida (os objectivos estratégicos ainda não estão totalmente promovidos e capacitados). No total, a janela temporal de onde foram retiradas as 9 sessões contempla 2 anos de terapia.

O passo seguinte passou pela visualização das sessões, acompanhada da respectiva cotação segundo o protocolo de análise e da recolha dos excertos do discurso da paciente que mais se salientaram (análogos aos excertos que nos lembramos quando estamos a resumir uma sessão ou a apresentá-la em supervisão). Estes excertos foram recolhidos pela primeira autora do estudo e a sua análise passou por diversas fases, geralmente em articulação com o segundo autor: 1) aprimoramento das definições dos processos incluídos no protocolo de cotação das sessões; 2) inclusão de novos processos, que os excertos sugeriam e não eram captados pelos processos já considerados; 3) atribuição de exemplos narrativos a cada processo definido; 4) ponderação da exclusão de processos pouco discriminatórios; 5) reflexão sobre possibilidades de os tornar mais discriminativos; 6) contagem dos processos identificados que possibilitassem comparações com os dados recolhidos através do protocolo de análise; 7) ponderação da categorização dos proces- 
sos em supra e sub-classes; 8) reenquadramento das fases e objectivos estratégicos das fases do meta-modelo como, respectivamente, supra e sub-classes de processos; 9) alocação dos excertos narrativos da paciente nas respectivas fases e objectivos estratégicos; 10) questionamento, a um primeiro perito, do seu acordo quanto à alocação dos excertos nas fases; 11) estimação do acordo entre avaliadores; 12) alteração das alocações conforme correcção do perito; 13) questionamento, de um segundo perito, do acordo relativo à alocação dos excertos nas fases, já com as correcções; 14) estimação do acordo entre os dois últimos avaliadores. $\mathrm{O}$ acordo entre a avaliadora e o $1^{\circ}$ juiz foi de $86 \%$ e o acordo entre os dois juízes foi de $100 \%$.

Como já foi referido, ao longo do estudo, foram-se estabelecendo como que duas frentes de trabalho: uma ligada ao reconhecimento e análise dos processos narrativos a nível molecular (a partir da revisão da literatura em narrativa, na base da formulação do protocolo de cotação), e outra a nível molar (a partir da teoria do MCP, no objecto da sua componente sequencial).

\section{Resultados}

Análise dos processos narrativos a nivel molecular

Na Figura 2 ilustram-se, com exemplos do discurso da paciente, os processos narrativos moleculares considerados.

Figura 2: Exemplos narrativos da paciente representativos dos processos narrativos considerados

\begin{tabular}{|l|}
\hline Abstracção \\
\hline $\begin{array}{l}\text { "Esta coisa de me sentir infantil é vulnerabilidade e é ser extremamente influen- } \\
\text { ciável pelo que os outros me dizem" }\end{array}$ \\
\hline Afirmação \\
\hline "Eu tenho muito medo de me expor" \\
\hline "Estou farta de ouvir o que os outros acham, quero ouvir o que eu tenho a dizer". \\
\hline Atribuição de significado \\
\hline "Senti que foi um descontrolo" \\
\hline "Isto foi para ocupar um espaço... agora é teimosia". \\
\hline Categorização \\
\hline "Se calhar é esta expectativa de estar sempre com capacidade de resposta" \\
\hline "Isso era estar protegida". \\
\hline Conflito \\
\hline $\begin{array}{l}\text { "Isto é muito confuso: não sentir nada e estar sempre à espera de sentir alguma } \\
\text { coisa" }\end{array}$ \\
\hline
\end{tabular}




\begin{tabular}{|c|}
\hline Consciencialização \\
\hline $\begin{array}{l}\text { "Eu não tinha esta noção, tinha a noção de que me preocupo muito com as coisas, } \\
\text { não tinha a noção que a nível emocional eu precisava tanto de não me expor" }\end{array}$ \\
\hline Contacto \\
\hline $\begin{array}{l}\text { "Isto não é bem um discurso, é uma coisa muito mais implícita, mas eu tenho isto } \\
\text { muito presente" }\end{array}$ \\
\hline Contemplação/observação \\
\hline $\begin{array}{l}\text { "Eu acho que estou com algumas possibilidades de coisas que gosto, mas ainda } \\
\text { não estou preparada para perder aquilo que eu tenho" }\end{array}$ \\
\hline Contextualização \\
\hline $\begin{array}{l}\text { "Pode ter muito a ver com o ano passado, em que eu estava a viver com o G e a A } \\
\text { e agora não tenho o dia muito preenchido" }\end{array}$ \\
\hline Descrição \\
\hline $\begin{array}{l}\text { "Cheguei lá e controlei-me um bocadinho. Estive a falar com uma colega, vamos } \\
\text { jantar no sábado" }\end{array}$ \\
\hline Diferenciação \\
\hline $\begin{array}{l}\text { "Parece que vou sempre com a fantasia de ser uma coisa muito boa e depois não } \\
\text { é" }\end{array}$ \\
\hline "Tudo é muito convicto nelas, eu estou no outro extremo" \\
\hline Elaboração \\
\hline $\begin{array}{l}\text { "Tenho estado a perceber a minha separação, e há dias que me parece muito } \\
\text { irreal" }\end{array}$ \\
\hline Especificação/concretização \\
\hline "Uma sensação imensa de angústia, de não conseguir respirar, que dói mesmo" \\
\hline Estabelecimento de relações \\
\hline $\begin{array}{l}\text { "Eu tenho estado a seleccionar um bocadinho os meus passos e acho que isso me } \\
\text { dá alguma energia" }\end{array}$ \\
\hline Exploração \\
\hline $\begin{array}{l}\text { "[Penso] onde é que isto me está a levar de facto, se é este mesmo o percurso que } \\
\text { eu quero fazer, onde é que eu quero chegar..." }\end{array}$ \\
\hline Generalização \\
\hline "Eu acho que nunca vou conseguir parar de procurar coisas" \\
\hline Responsabilização \\
\hline "Mas não é a questão delas, é a minha questão, o facto de eu precisar tanto..." \\
\hline
\end{tabular}

Da análise descritiva dos processos identificados nas sessões visualizadas, surgiram algumas diferenças que vale a pena referir.

As médias e os desvios-padrão dos processos narrativos para a amostra total, sugerem que a paciente utiliza, preferencialmente, os processos narrativos de elaboração $(\mathrm{M}=6.89, \mathrm{DP}=1.05)$, exploração $(\mathrm{M}=6.56, \mathrm{DP}=1.42)$, consciencialização $(\mathrm{M}=6.33, \mathrm{DP}=1.00)$, e diferenciação $(\mathrm{M}=6.22, \mathrm{DP}=1.72)$. 
Tabela 1: Análise descritiva dos processos narrativos para as fases predominantes 2,3 e 4

\begin{tabular}{|c|c|c|c|c|c|c|c|c|c|c|c|c|c|c|c|}
\hline & \multicolumn{5}{|c|}{ Fase pred. 2} & \multicolumn{5}{|c|}{ Fase pred. 3} & \multicolumn{5}{|c|}{ Fase pred. 4} \\
\hline & $\mathrm{N}$ & Min. & Máx. & $\mathrm{M}$ & DP & & in. & Máx. & M & $\mathrm{DP}$ & $\mathrm{N}$ & Min. & Máx. & M & $\mathrm{DP}$ \\
\hline Elaboração & 3 & 5 & 8 & 6.33 & 1.53 & 3 & 6 & 7 & 6.67 & .58 & 3 & 7 & 8 & 7.67 & .58 \\
\hline Exploração & 3 & 4 & 6 & 5.33 & 1.15 & 3 & 7 & 8 & 7.33 & .58 & 3 & 5 & 8 & 7.00 & 1.73 \\
\hline Consciencialização & 3 & 6 & 8 & 7.00 & 1.00 & 3 & 5 & 7 & 6.00 & 1.00 & 3 & 5 & 7 & 6.00 & 1.00 \\
\hline Diferenciação & 3 & 3 & 6 & 4.33 & 1.53 & 3 & 7 & 7 & 7.00 & .00 & 3 & 6 & 8 & 7.33 & 1.15 \\
\hline Est. de relações & 3 & 3 & 5 & 4.33 & 1.15 & 3 & 3 & 7 & 5.33 & 2.08 & 3 & 5 & 8 & 6.33 & 1.53 \\
\hline Generalização/Abstr. & 3 & 3 & 5 & 4.00 & 1.00 & 3 & 3 & 5 & 4.33 & 1.15 & 3 & 5 & 7 & 6.33 & 1.15 \\
\hline Descrição & 3 & 2 & 6 & 4.33 & 2.08 & 3 & 4 & 6 & 5.00 & 1.00 & 3 & 4 & 6 & 5.00 & 1.00 \\
\hline Especificação/Concr. & 3 & 5 & 6 & 5.33 & .58 & 3 & 4 & 5 & 4.33 & .58 & 3 & 4 & 4 & 4.00 & .00 \\
\hline Atr. de significado & 3 & 3 & 5 & 3.67 & 1.15 & 3 & 1 & 6 & 3.00 & 2.65 & 3 & 3 & 7 & 4.67 & 2.08 \\
\hline Contextualização & 3 & 2 & 6 & 4.33 & 2.08 & 3 & 3 & 3 & 3.00 & .00 & 3 & 1 & 3 & 2.33 & 1.15 \\
\hline Categorização & 3 & 1 & 2 & 1.67 & .58 & 3 & 1 & 5 & 2.67 & 2.08 & 3 & 2 & 3 & 2.67 & .58 \\
\hline Responsabilização & 3 & 2 & 3 & 2.67 & .58 & 3 & 0 & 3 & 1.33 & 1.52 & 3 & 2 & 4 & 3.00 & 1.00 \\
\hline Afirmação & 0 & & & & & 2 & 0 & 2 & 1.00 & 1.41 & 3 & 1 & 5 & 2.33 & 2.31 \\
\hline
\end{tabular}


A análise das amostras segundo as 3 fases consideradas denota, no entanto, que há alguns processos que apresentam valores elevados especialmente numa ou duas delas, em comparação com os restantes processos narrativos na mesma fase: na fase predominante 2 destaca-se o processo especificação/concretização $(\mathrm{M}=5.33, \mathrm{DP}=.58)$, na fase predominante 3 salienta-se o processo narrativo estabelecimento de relações $(\mathrm{M}=5.33, \mathrm{DP}=2.08)$, e na fase predominante 4 elevam-se os processos estabelecimento de relações $(\mathrm{M}=6.33, \mathrm{DP}=1.53)$ e generalização/abstracção $(\mathrm{M}=6.33, \mathrm{DP}=1.15)$. Testes t-student de comparação de médias não indicaram, contudo, que as diferenças entre as fases consideradas fossem significativas.

De forma a tornar a contabilização de cada processo narrativo em cada sessão menos inferencial, a cada excerto recolhido do discurso da paciente foram atribuídos os processos narrativos representados e estabelecida a percentagem de cada processo no total dos processos por fase predominante. A Figura 3 dá conta das diferenças nas percentagens dos diversos processos narrativos entre as diversas fases.

Figura 3: Comparação das percentagens dos processos narrativos 1 nas três fases predominantes

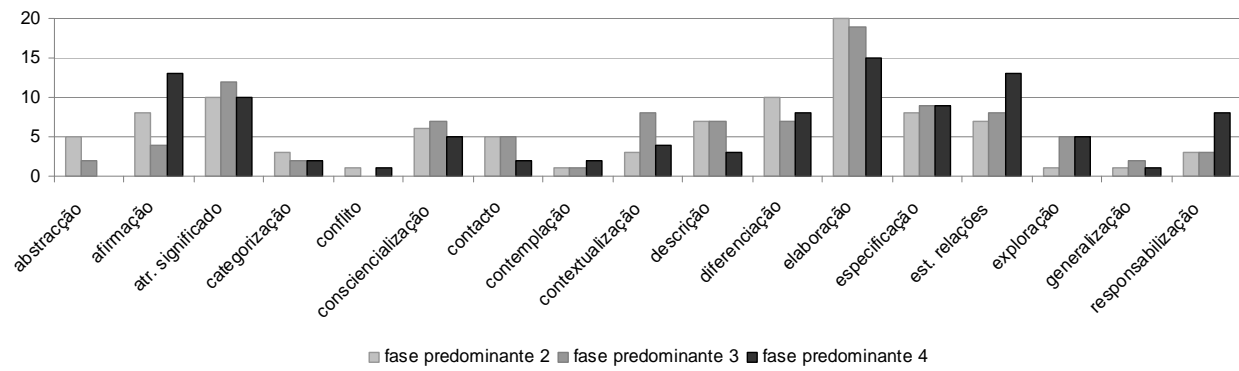

Nesta figura, já se denotam elevações não encontradas nos resultados obtidos em função da análise do protocolo de cotação, nomeadamente, a maior elevação do processo diferenciação na fase predominante 2 , mais descrição nas fases predominantes 2 e 3 e menos na fase predominante 4, um ligeiro destaque do processo atribuição de significado e um grande destaque do processo contextualização na fase predominante 3 , e a saliência dos processos afirmação e responsabilização na fase predominante 4.

\footnotetext{
${ }^{1}$ Identificados na análise dos excertos do discurso da paciente
} 


\section{Análise dos processos narrativos a nível molar}

Os excertos narrativos da paciente foram alocados aos objectivos estratégicos que representam e a Figura 4 ilustra, com exemplos do discurso da paciente, os objectivos estratégicos das três fases consideradas.

Figura 4: Exemplos narrativos da paciente representativos dos objectivos estratégicos das fases consideradas

\begin{tabular}{|c|c|}
\hline \multirow{10}{*}{ 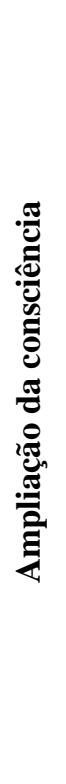 } & $\begin{array}{l}\text { Sentir curiosidade e interesse em observar as formas como se trata a si } \\
\text { mesma }\end{array}$ \\
\hline & $\begin{array}{l}\text { "Eu acho que estou com muita dificuldade ou muito medo de sentir seja o que } \\
\text { for... não sei, ocorreu-me agora isto..." }\end{array}$ \\
\hline & $\begin{array}{l}\text { Explorar ou experienciar o impacto que situações relevantes têm em si } \\
\text { própria }\end{array}$ \\
\hline & $\begin{array}{l}\text { "Quando estou ao pé dele tenho a sensação de ser muito infantil, de precisar } \\
\text { muito dele" }\end{array}$ \\
\hline & $\begin{array}{l}\text { Ampliar a consciência das formas como ela se relaciona com os outros e } \\
\text { os outros com ela }\end{array}$ \\
\hline & $\begin{array}{l}\text { "As linhas estão pouco definidas entre nós, e eu não percebo muito bem o que } \\
\text { se passa entre nós" }\end{array}$ \\
\hline & $\begin{array}{l}\text { Traduzir experiências problemáticas nos seus elementos (cognição, emo- } \\
\text { ção, comportamento) }\end{array}$ \\
\hline & $\begin{array}{l}\text { "Uma sensação imensa de angústia, de não conseguir respirar, que dói mes- } \\
\text { mo" }\end{array}$ \\
\hline & Tomar consciência de partes ou necessidades de si própria em conflito \\
\hline & $\begin{array}{l}\text { "Isto é muito confuso: não sentir nada e estar sempre à espera de sentir } \\
\text { alguma coisa" }\end{array}$ \\
\hline \multirow{8}{*}{ 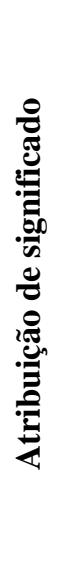 } & Identificar padrões existentes nos seus modos habituais de funcionamento \\
\hline & "A sensação que tinha era que estava desde sempre sozinha" \\
\hline & $\begin{array}{l}\text { Superar os processos que dificultam a consciência da experiência e a } \\
\text { construção de significados reparadores }\end{array}$ \\
\hline & $\begin{array}{l}\text { "Nem eu via nada em mim [no passado], eu achava que tinha que estar ali, } \\
\text { que tinha que estar a aguentar" }\end{array}$ \\
\hline & $\begin{array}{l}\text { Formular novas ligações ou explicações plausíveis para as suas atitudes } \\
\text { ou comportamento problemáticos }\end{array}$ \\
\hline & $\begin{array}{l}\text { "Reparei o quanto vou lá por causa da minha mãe, pelo quanto ela faz ques- } \\
\text { tão, precisa de nós" }\end{array}$ \\
\hline & $\begin{array}{l}\text { Compreender o que procura alcançar quando age de formas que acabam } \\
\text { por lhe criar dificuldades }\end{array}$ \\
\hline & $\begin{array}{l}\text { "O ir lá é encontrar qualquer coisa que é muito confortável para mim, e } \\
\text { nunca é" }\end{array}$ \\
\hline
\end{tabular}




\begin{tabular}{|c|c|}
\hline & $\begin{array}{l}\text { Perceber as suas experiências problemáticas à luz das circunstâncias do } \\
\text { passado ou do presente }\end{array}$ \\
\hline & "No sítio onde nasci e cresci há muita obrigação" \\
\hline \multirow{10}{*}{ 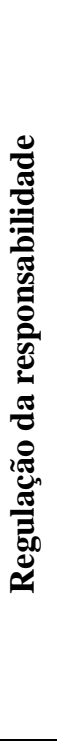 } & $\begin{array}{l}\text { Assumir responsabilidade por cuidar de si, experimentando mobilizar } \\
\text { recursos internos ou externos nesse sentido }\end{array}$ \\
\hline & "Estou a tentar ver o que é que me faz mais sentido, me preenche mais" \\
\hline & $\begin{array}{l}\text { Assumir o compromisso por respeitar e validar as suas necessidades, } \\
\text { quer elas correspondam, ou não, às expectativas dos outros }\end{array}$ \\
\hline & $\begin{array}{l}\text { "Se para mim é importante, porque é que eu hei-de ter aquela vida que os } \\
\text { outros acham que eu devo ter" }\end{array}$ \\
\hline & Reconhecer que só ela é o agente activo das suas próprias escolhas \\
\hline & $\begin{array}{l}\text { "Se eu não sei muito bem onde isto me está a levar porque é que eu não hei- } \\
\text {-de fazer outra coisa" }\end{array}$ \\
\hline & $\begin{array}{l}\text { Compreender quando e como é que ela próprio contribui para as suas } \\
\text { dificuldades habituais }\end{array}$ \\
\hline & $\begin{array}{l}\text { "Parece que eu inicio uma coisa e vou e nunca chego a sentir aquela vibra- } \\
\text { ção e ver aonde aquilo leva" }\end{array}$ \\
\hline & $\begin{array}{l}\text { Perceber que é ela que pode promover ou dificultar a satisfação das suas } \\
\text { necessidades }\end{array}$ \\
\hline & $\begin{array}{l}\text { "Eu acho que estou com algumas possibilidades de coisas que gosto, mas } \\
\text { ainda não estou preparada para perder aquilo que eu tenho" }\end{array}$ \\
\hline
\end{tabular}

A distribuição dos excertos pelas três fases do MCP permitiu clarificar os processos narrativos dentro de cada fase e entre fases predominantes. Não havendo homogeneidade na quantidade de excertos recolhidos nas 9 sessões, e de forma a efectuarmos uma comparação mais realista entre as fases predominantes, estabelecemos a percentagem de excertos representativos de cada fase em função do total dos excertos recolhidos em cada sessão. A Figura 5 dá conta das diferenças a nível de processos narrativos dentro de cada fase predominante e entre fases.

A distribuição sugere que, para cada conjunto de três sessões (correspondentes às fases predominantes), há narrativas mais representativas de uma ou duas fases: no primeiro conjunto as narrativas são especialmente representativas das fases dois e três, no segundo conjunto as narrativas são mais representativas das fases dois e três, com a fase três cada vez mais sólida, e no terceiro conjunto as narrativas são mais representativas da fase quatro. Paralelamente, exceptuando para as sessões da fase predominante 2 (já em transição para a fase três), a distribuição sugere que quando há trabalho predominante de uma fase, a fase precedente ainda está bastante activa e a fase posterior ainda é praticamente inexistente. As sessões de fase predominante 4 sugerem, ainda, que se a fase imediatamente precedente ainda está bastante activa, a fase anterior a esta última já está bastante desvanecida. 
Figura 5: Diferenças nos processos narrativos intra e inter-fases $(\%)^{2}$

\begin{tabular}{|c|c|c|c|c|}
\hline & & \multicolumn{3}{|c|}{ Narrativas } \\
\hline & & Fase 2 & Fase 3 & Fase 4 \\
\hline \multirow{3}{*}{ 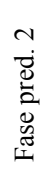 } & Sessão 31 & 71 & 17 & 12 \\
\hline & Sessão 36 & 46 & 54 & 0 \\
\hline & Sessão 46 & 34 & 45 & 21 \\
\hline \multirow{3}{*}{ 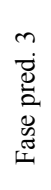 } & Sessão 51 & 57 & -29 & 14 \\
\hline & Sessão 65 & 44 & 56 & 0 \\
\hline & Sessão 80 & 48 & 48 & $\| 4$ \\
\hline \multirow{3}{*}{ 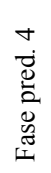 } & Sessão 90 & 34 & 37 & 29 \\
\hline & Sessão & 0 & 17 & 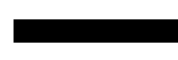 \\
\hline & 100 & 11 & 40 & 53 \\
\hline
\end{tabular}

\section{Discussão dos resultados}

Processos narrativos a nível molecular

A coincidência das elevações, na amostra total e nas três fases predominantes, dos processos elaboração, exploração, consciencialização e diferenciação, não permite retirar ilações quanto aos movimentos destes processos entre as fases. Ao nível da análise do discurso da paciente notou-se maior elevação do processo diferenciação na fase predominante 2 , de acordo com o esperado à luz do modelo, mas a diferença de elevação com as fases seguintes é, contudo, reduzida. Esta transversalidade (nos quatro processos) pode dever-se a: 1) os processos serem característicos do estilo de funcionamento da paciente e não resultado do trabalho terapêutico; 2) os processos terem sido conquistados na fase 2 (ou mesmo na fase 1) e mantidos nas fases

\footnotetext{
${ }^{2}$ A soma das percentagens de narrativas para cada sessão é, por vezes, superior a 100, uma vez que os mesmos excertos narrativos corresponderam, algumas vezes, a mais do que uma fase e/ou objectivos estratégicos.
} 
posteriores; 3) os processos serem, de facto, transversais às diferentes fases e/ou a diferentes pacientes; ou 4) os processos estarem pouco discriminados e, consequentemente, encaixarem nas diferentes fases, embora materializando-se de formas diferentes. Apesar da transversalidade destes processos, eles podem ter funções diferentes em cada fase predominante, como a análise dos processos a nível molar sugere (referir-nos-emos a esta mais à frente).

Quanto aos processos que permitiram fazer diferenciações, o destaque do processo especificação/concretização na fase predominante 2 é congruente com os objectivos estratégicos da fase, relacionados com a ampliação da consciência da experiência e do self, apelando à necessidade de concretizar/analisar a realidade subjectiva de cada um. Para além disso, é nesta fase que se começa a procurar mais activamente o acesso e a partilha de acontecimentos autobiográficos concretos, em vez de acontecimentos episódicos gerais ou inespecíficos, acreditando que é no concreto e específico que está a experiência, o impacto do meio, e a interpretação que a pessoa faz de ambos (Greenberg et al., 1993). A não elevação deste processo na fase predominante 3 é também congruente com os objectivos estratégicos desta fase, que se prendem com a elevação no nível de abstracção para perceber a ligação entre vários elementos. Por outro lado, a elevação deste processo na fase predominante 4, sugerida pela análise dos excertos do discurso da paciente, não deixa de ser coerente, uma vez que o detalhe, o pormenor, a subtileza das escolhas ou possibilidades trabalhadas nesta fase pode fazer elevar esse processo.

Por outro lado, o relevo do processo de estabelecimento de relações na fase predominante 3 é também congruente com os objectivos estratégicos da fase, relacionados com a construção de novos significados relativos à experiência e ao self. Facilmente se reconhece que a atribuição de significado implica estabelecimento de relações. Contudo, comparações entre fases revelam que este processo não é particular a esta fase, elevando-se, também, na fase predominante 4.

Nesta última fase considerada, destacam-se os processos estabelecimento de relações e generalização/abstracção. A análise dos objectivos estratégicos da fase quatro revela que a compreensão, por parte do paciente, do seu papel nos seus problemas, ou nas soluções para estes, implica estabelecimento de relações, e o reconhecimento da sua responsabilidade e o compromisso por respeitar as suas necessidades, implicam abstracção. Por outro lado, lembrando que o estabelecimento de relações se eleva também na fase predominante 3 , esta elevação pode, adicionalmente, reflectir a consolidação das conquistas de fase três.

Conceptualmente alocados à fase predominante 4 estão os processos afirmação e responsabilização, com valores baixos ao nível dos resultados da cotação do protocolo. Lembrando, contudo, que a paciente ainda se encontra em fase predominante 4 , não tendo capacidade robusta nesta fase, 
os resultados podem reflectir que as conquistas terapêuticas da fase quatro ainda não estão asseguradas. Por outro lado, os dados decorrentes da análise dos excertos do discurso da paciente revelaram resultados animadores relativamente aos processos afirmação e responsabilização nesta fase, tendo, estes, elevado bastante em comparação com as fases predominantes anteriores. A análise mais cuidada dos excertos das sessões poderá ter revelado capacidades mais subtis, que a cotação inferencial, imediatamente após as sessões, deixou escapar.

Relativamente ao processo descrição, a transversalidade sugerida pelos resultados da análise do protocolo faz sentido à luz da visão do processo terapêutico como um espaço de revelação/partilha. A sugestão, a partir da análise do discurso da paciente, de diminuição gradual deste processo ao longo das três fases predominantes, pode estar relacionada, não com a sua extinção gradual, mas com a perda de primazia, comedindo-se aos bastidores de processos mais reflexivos.

Também ao nível da análise dos excertos narrativos, o destaque acentuado do processo contextualização na fase predominante 3 está de acordo com o sugerido pelo modelo, uma vez que a construção de novos significados relativos à experiência e ao Self implica perceber as ligações contextuais que acompanham os problemas em foco; e por último, o ligeiro destaque do processo atribuição de significado na fase predominante 3 está claramente de acordo com o modelo, como o próprio nome da fase indica.

\section{Processos narrativos a nível molar}

A distribuição dos excertos narrativos da paciente pelas três fases do meta-modelo consideradas dá conta de movimentação entre sessões, no sentido de, progressivamente, a paciente reconstruir as suas narrativas na sequência proposta pelo modelo, pelo menos entre as fases dois e quatro. Esta progressão sequencial das narrativas vai, também, ao encontro do paradigma organísmico-desenvolvimentista, referido por Achenbach (1986), que defende que o desenvolvimento humano é sequencial, em que níveis mais avançados estão dependentes da emergência de níveis menos avançados, e consolida a componente sequencial do MCP.

Apesar da movimentação verificada, as fases predominantes 2 e 3 contêm muitas narrativas uma da outra. Estudos anteriores têm mostrado que o trabalho efectuado nunca é puramente de uma fase mas que há frequentes sobreposições, particularmente entre fases adjacentes, como é o caso. Ressalva-se que quanto mais sobreposição entre fases adjacentes maior a probabilidade de estarmos perante uma fase de transição, pelo que podemos ter vindo, curiosamente, encontrar um conjunto de sessões que, consideradas sessões de transição, poderão ser alvo de investigações muito interessantes 
para estudar os processos de transição de fase (Piaget, 1977; Raijmakers \& Molenaar, 2004).

A revelação que, em termos narrativos, para este caso e nestas três fases predominantes, a fase anterior à fase predominante ainda está muito activa mas a fase anterior a esta última já está muito desvanecida, faz pensar no conceito gestalt figura-fundo, em que os ganhos de fases precedentes se vão tornando cada vez mais fundo, permitindo que os ganhos da fase predominante se tornem cada vez mais figura. No mesmo sentido, a análise qualitativa dos excertos permitiu perceber que se, por um lado, o foco avança naturalmente ao longo das fases, a análise mais cuidada dos excertos das sessões em fase predominante 4 revela que os processos de ampliação de consciência (fase dois) estão lá, só que muito mais ao serviço do reconhecimento das próprias necessidades e responsabilidade.

\section{Conclusão}

No geral, os resultados do estudo apontam para diferenciação dos processos narrativos ao longo do processo terapêutico. A análise dos processos moleculares não permitiu, contudo, retirar um padrão. Com o intuito de promover uma organização/estrutura para o processamento narrativo ao longo da terapia, recorremos à teoria da componente sequencial de fases do MCP para formular novas categorias, também elas ancoradas em processos mais do que em conteúdos, que representam as fases predominantes contempladas nas sessões em estudo, a fase dois, a três e a quatro respectivamente. Tratando-se de categorias mais molares, já permitiram inferir um padrão mais claro de movimento narrativo, funcionando como uma heurística interpretativa da diferenciação e movimentação narrativa ao longo do processo terapêutico. Para além disso, este dado constitui evidência longitudinal, na voz de uma (primeira) paciente, que corrobora a sequência proposta pela componente sequencial do meta-modelo, complementando os dados quantitativos transversais recolhidos em estudos anteriores.

Adicionalmente, o estudo veio evidenciar a pertinência de articular o MCP com modelos como o de Processo Narrativo (Angus et al., 1999) e de Assimilação (Stiles et al., 1990, 1991). Por um lado, o MCP complementa o modelo de Angus et al. (1999), na medida em que especifica capacidades narrativas sequenciais ao longo do processo terapêutico que o Modelo de Processo Narrativo não discrimina; e por outro complementa o Modelo de Assimilação (Stiles et al., 1990, 1991), na medida em que considera processos mais molares, menos momento-a-momento (fases e objectivos estratégicos) que permite maior articulação com as macro-narrativas dos pacientes. A introdução de uma reflexão ao nível dos objectivos estratégicos mais gerais, 
a um nível fase-a-fase, em complemento à ponderação do trabalho clínico a realizar momento-a-momento, pode ser muito produtiva ao nível da prática clínica, contexto em que é essencial não nos perdermos em aspectos muito particulares, negligenciando o panorama mais abrangente, nem focarmo-nos exclusivamente nas circunstâncias gerais, descurando as suas particularidades.

Adicionalmente, o MCP é bastante abrangente e integrativo, contemplando processos emocionais, cognitivos, interpessoais, motivacionais, comportamentais e organísmicos, baseados em factores comuns, de que dá conta nos objectivos estratégicos das fases. Esta abrangência é de extrema importância para a construção de narrativas mais coerentes e que permitam a regulação da satisfação de necessidades psicológicas, pois apesar da coerência narrativa ser um grande objectivo terapêutico, procurá-la apenas com métodos narrativos pode ser insuficiente.

O MCP mostrou, ainda, ser promissor no estudo da mudança narrativa em psicoterapia.

Apesar das potencialidades apontadas, várias limitações surgiram no caminho, algumas para cuja minimização o próprio estudo veio dar o seu contributo, outras que é importante que estudos posteriores venham colmatar.

O enfoque nos processos narrativos, dentre a vastidão de processos e comportamentos que influenciam e demonstram a capacidade dos indivíduos, não permite análises tão holísticas como desejado da mudança nos pacientes, embora o presente estudo pretenda ser já um pequeno passo possibilitador de maiores investimentos futuros.

A selecção e definição dos processos considerados no estudo foi, também, pouco sustentada na literatura e investigação na área, pelo que beneficiariam de pesquisas mais cuidadas. A falta de material audiovisual que contemple as sete fases do modelo e a dificuldade de garantir sessões absolutamente representativas de uma única fase constituiu uma outra limitação.

$\mathrm{O}$ recurso ao próprio terapeuta como um segundo avaliador, apesar de várias vantagens, traz também limitações ao nível da falta de neutralidade.

A recolha de excertos, em vez da transcrição das sessões, limitou, também, as possibilidades de análise e comparação dos dados, uma vez que as narrativas mais salientes não são, necessariamente, as mais frequentes.

$\mathrm{O}$ presente estudo veio mostrar, contudo, o potencial do MCP como uma ferramenta de análise e de conceptualização da evolução dos pacientes (e das suas necessidades) através das próprias narrativas, bem como um auxiliar da tomada de decisão clínica.

O investimento na análise longitudinal de um estudo de caso, foi uma grande aposta do estudo, com implicações produtivas em diversas áreas. $\mathrm{Na}$ prática clínica tem implicações ao nível da avaliação das capacidades e vul- 
nerabilidades do paciente, e do favorecimento da tomada de decisão clínica quanto ao avanço, manutenção e recuo na prossecução dos objectivos estratégicos. Na formação/treino e supervisão à luz do modelo, favorece a disponibilização de exemplos mais precisos e ao nível concreto do discurso dos pacientes do que promover ou atender neles em termos narrativos. Ao nível da teoria psicológica e psicoterapêutica, traz a nova corroboração da sequencialidade do modelo e especificação dos objectivos estratégicos ao longo do processo terapêutico em termos narrativos. Por último, favorece a investigação, abrindo portas para a integração de conhecimentos e novas vias de investigação, no sentido do aprofundamento do tema.

A este nível da investigação, parece-nos pertinente analisar outros casos clínicos e em mais fases da componente sequencial do meta-modelo, averiguando a replicação dos resultados no sentido de permitir generalizações, e continuar a reflectir sobre supra e sub-categorias de processos narrativos, independentes das fases do meta-modelo, de forma a aprimorar os próprios objectivos estratégicos das fases e contribuir para conceptualizações teóricas do desenvolvimento narrativo do adulto no processo terapêutico.

\section{Referências}

Achenbach, T. M. (1986). The developmental study of psychopathology: Implications for psychotherapy and behavior change. In S. L. Garfield \& A. E. Bergin (Eds.), Handbook of psychotherapy and behavior change ( $3^{\mathrm{a}}$ ed.) (pp. 117-154). New York, NY: John Wiley.

Angus, L., Bryntwick, E., Boritz, T., Warwar, S., \& Greenberg, L. (2008, Junho). Integrating expressed emotion with autobiographical memory specificity in effective treatments of depression: An intensive theme analysis of key shift events. Comunicação apresentada no 39th International Meeting of the Society for Psychotherapy Research. Barcelona, Espanha.

Angus, L.; Levitt, H.; \& Hardtke, K. (1999). The narrative processes coding system: Research applications and implications for psychotherapy practice. Journal of clinical psychology, 55(10), 1255-1270.

Angus, L. E., Lewin, J., Bouffard, B., \& Rotondi-Trevisan, D. (2004). "What's the story?" Working with narrative in experiential psychotherapy. In L. E. Angus \& J. McLeod (Eds.), The handbook of narrative and psychotherapy: Practice, theory and research (pp. 87-102). Thousand Oaks, CA: Sage.

Baptista, T. V. (2000). The effectiveness of psychotherapy in changing life's narratives (Tese de doutoramento não publicada, Faculdade de Psicologia, Lisboa, Portugal).

Battro, A. M. (1978). Dicionário terminológico de Jean Piaget. São Paulo, Brasil: Livraria Pioneira.

Beutler, L. E., Consoli, A., \& Lane, G. (2005). Systematic treatment selection and prescritive psychotherapy. In J. C. Norcross, \& M. R. Goldfrield (Eds.), Hand- 
book of psychotherapy integration (2 $2^{\mathrm{a}}$ ed.) (pp. 121-143). New York, NY: Oxford.

Conceição, N. M. (2005). Articulação entre promoção e assimilação de objectivos estratégicos: Marcadores para a sequencialização temporal do processo psicoterapêutico (Dissertação de mestrado não publicada, Faculdade de Psicologia, Lisboa, Portugal).

Conceição, N. M. (2010). If you want to get ahead, get a sequence of general strategies: Integrative decision making in real-world psychotherapy (Tese de doutoramento não publicada, Faculdade de Psicologia, Lisboa, Portugal).

Conceição, N., \& Vasco, A. (2008). Paradigmatic complementarity strategic objectives manual, Version 4.0 (Manuscrito não publicado, Faculdade de Psicologia, Universidade de Lisboa, Portugal).

Conceição, N., \& Vasco, A. B. (2009, Maio). To live effectively is to live with adequate information: Longitudinal case-analyses of the use of strategic objectives in paradigmatic complementarity. Comunicação apresentada na XXV International Conference of the Society for the Exploration of Psychotherapy Integration. Seatle, E.U.A.

Detert, N. B., Llewelyn, S., Hardy, G. E., Barkham, M., \& Stiles, W. B. (2006). Assimilation in good- and poor-outcome cases of very brief psychotherapy for mild depression: An initial comparison. Psychotherapy Research, 16(4), 393-407 .

Gold, J. R. (1994). When the patient does the integration: Lessons for theory and practice. Journal of Psychotherapy Integration, 4, 133-158.

Gonçalves, I., \& Vasco, A. B. (2001). Estudo de caso de uma perturbação "borderline da personalidade" à luz do modelo de "complementaridade paradigmática". Psicologia, 15(2), 227-266.

Gonçalves, O. F., Henriques, M. R., \& Machado, P. P. (2004). Nurturing nature: Cognitive narrative strategies. In L. E. Angus \& J. McLeod (Eds.), The Handbook of Narrative and Psychotherapy: Practice, theory and research (pp. 103-118). Thousand Oaks, CA: Sage.

Greenberg, L. S., \& Pascual-Leone, J. (2001). A dialectical constructivist view of the creation of personal meaning. Journal of Constructivist Psychology, 14, 165-186.

Greenberg, L. S., Rice, L. N., \& Elliott, R. (1993). Facilitating emotional change: The moment-by-moment process. New York, NY: Guilford Press.

Hardtke, K. K., \& Angus, L. E. (2004). The narrative assessment interview: Assessing self-change in psychotherapy. In L. E. Angus \& J. McLeod (Eds.), The handbook of narrative and psychotherapy: Practice, theory and research (pp. 247-262). Thousand Oaks, CA: Sage.

Honos-Webb, L., Stiles, W. B., \& Greenberg, L. S. (2003). A method of rating assimilation in psychotherapy based on markers of change. Journal of Counseling Psychology, 50, 189-198.

Lambert, M. J., \& Bergin, A. E. (1994). Achievements and limitations of psychotherapy research. In D. F. Freedheim (Ed.), History of psychotherapy: A century of change (pp. 360-390). Washington, DC: APA. 
Lazarus, A. A. (2005). Multimodal therapy. In J. C. Norcross, \& M. R. Goldfrield (Eds.), Handbook of psychotherapy integration (2a ed.) (pp. 105-120). New York, NY: Oxford.

Lourenço, O. (2005). Psicologia de desenvolvimento cognitivo (2a ed.). Coimbra, Portugal: Almedina.

Moleiro, C. (2005). Sobre o/a cliente e para além do diagnóstico: Contributos para a optimização da tomada de decisão clínica. Psychologica, 40, 37-53.

Moreira, P., Beutler, L. E., \& Gonçalves, O. F. (2008). Narrative change in psychotherapy: Differences between good and bad outcome cases in cognitive, narrative, and prescriptive therapies. Journal of Clinical Psychology, 64(10), 1181-1194 .

Norcross, J. C. (2005). A primer on psychotherapy integration. . In J. C. Norcross, \& M. R. Goldfrield (Eds.), Handbook of psychotherapy integration (pp. 3-23). New York, NY: Oxford University Press.

Osatuke, K., Glick, M. J., Gray, M. A., Reynolds, D. J. Jr., Humphreys, C. L., Salvi, L. M., \& Stiles, W. B. (2004). Assimilation and narrative: Stories as meaning bridges. In L. E. Angus \& J. McLeod (Eds.), The Handbook of Narrative and Psychotherapy: Practice, theory and research (pp. 193-210). Thousand Oaks, CA: Sage.

Piaget, J. (1977). O desenvolvimento do pensamento: Equilibração das estruturas cognitivas. Lisboa, Portugal: Dom Quixote.

Prochaska, J. O., \& DiClemente, C. (2005). The transtheoretical approach. In J. C. Norcross, \& M. R. Goldfrield (Eds.), Handbook of psychotherapy integration ( $2^{\mathrm{a}}$ ed.) (pp. 147-171). New York, NY: Oxford.

Raijmakers, M. E., \& Molenaar, P. C. (2004). Modeling developmental transitions in adaptive reasonance therory. Developmental Science, 7(2), 149-157.

Rudolf, G., Grande, T., Dilg, R., Jakobsen, T., Keller, W., Oberbracht, C., Pauli-Magnus, C., Stehle, S., \& Wikle, S. (2002). Strucutural changes in psychoanalytic therapies - The Heidelberg-Berlin study on long-term psychoanalytic therapies (PAL). In M. Leuzinger-Bohleber \& M. Target (Eds.), Longer term psychoanalytic treatment - Perspectives for therapists and researchers (pp. 201-222). London, England: Whurr.

Shrout, P. E. \& Fleiss, J. L. (1979) Intraclass correlations: Uses in assessing rater reliability. Psychological Bulletin, 2, 420-428.

Stiles, W. B., Elliott, R., Llewelyn, S. P., Firth-Cozens, J. A., Margison, F. R., Shapiro, D. A., \& Hardy, G. (1990). Assimilation of problematic experiences by clients in psychotherapy. Psychotherapy, 27, 411-420.

Stiles, W.B., Honos-Wehb, L., \& Lani, J.A. (1999). Some functions of narrative in the assimilation of problematic experiences. Journal of Clinical Psychology, $55,1213-1226$

Stiles, W. B.; Morrison, L. A.; Haw, S. K.; Harper, H.; Shapiro, D. A.; \& Firth-Cozens, J. (1991). Longitudinal study of assimilation in exploratory psychotherapy. Psychotherapy: Theory, Research, Practice, Training, 28(2), 195-206. 
Tallman, K., \& Bohart, A. C. (1999). The client as a common factor: Clients as self-healers. In M. A. Hubble, B. L. Duncan, \& S. D. Miller (Eds.), The heart and soul of change (pp. 91-131). Washington, DC: APA.

Toukmanian, S. G. (1986). A measure of client perceptual processing. In L. S. Greenberg, \& W. M. Pinsof (Eds.), The psychotherapeutic process: A research handbook (pp. 107-130). New York, NY: Guilford Press.

Vasco, A. B. (2001). Fundamentos para um modelo integrativo de "complementaridade paradigmática". Psicologia, 15, 219-226.

Vasco, A. B. (2006). Entre nuvens e relógios: Sequência temporal de objectivos estratégicos e integração em psicoterapia. Revista Portuguesa de Psicologia, 39, 9-31. 
APÊNDICE A

5. reconhecer que é capaz de lidar de forma autónoma com os futuros desafios da vida
4. antecipar recursos para lidar com cenários futuros para além da conclusão do processo terapêutico

7 3. projectar-se no futuro efectiva e afectivamente relacionado consigo próprio(a), com os outros e com o mundo

1. fortalecer a sensação de coerência pessoal e de que a sua vida tem sentido como um todo

5. generalizar a expressão da sua identidade nas diferentes áreas da sua vida

4. cuidar-se emocionalmente no que respeita à expressão da sua identidade e crescimento

6 3. aceitar a inevitabilidade de um certo grau de vulnerabilidade ou conflito na vivência e expressão da sua identidade 2. desenvolver ou fortalecer relações/situações que apoiem as suas escolhas

1. lidar com obstáculos internos ou externos à consolidação da sua identidade e crescimento

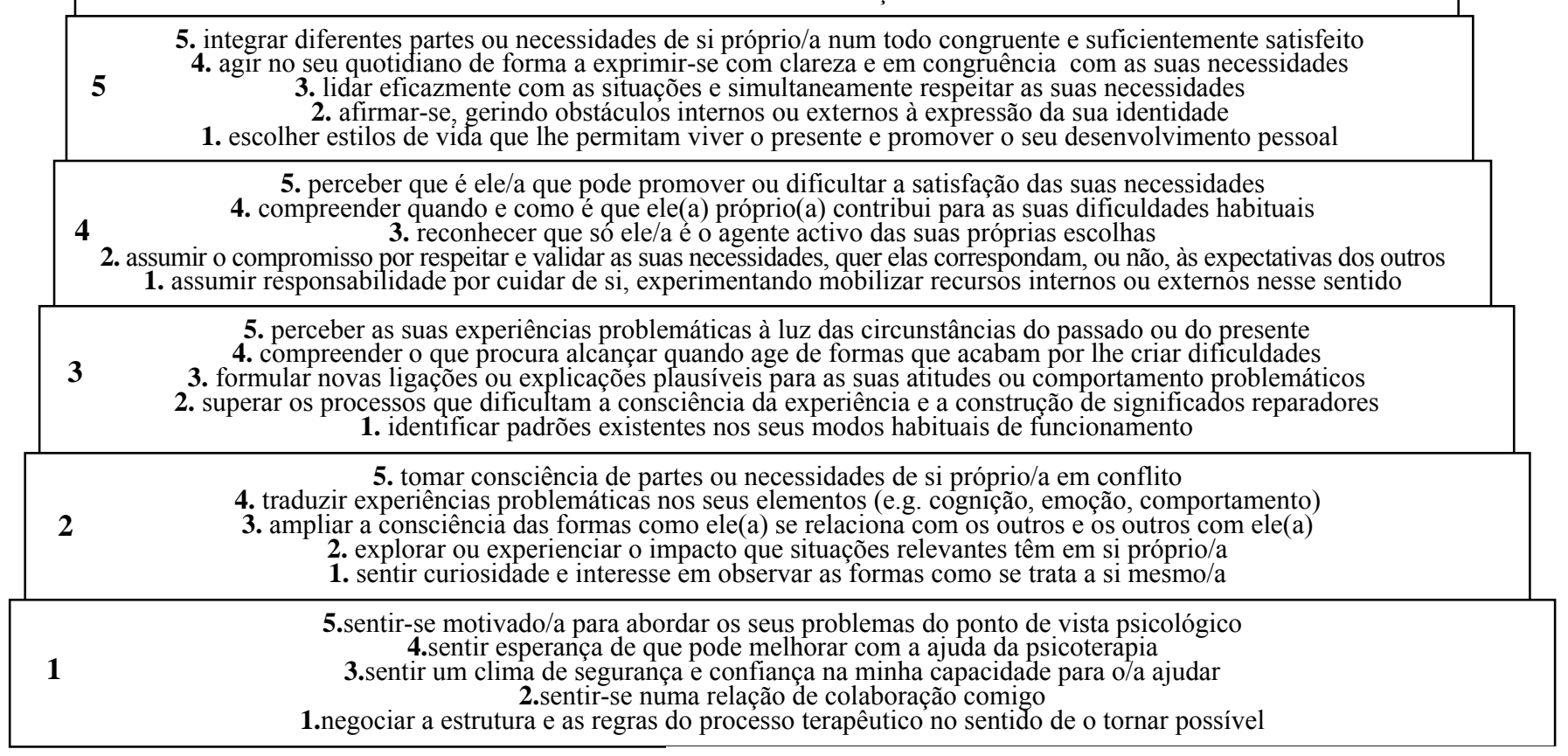

๑) Complementaridade Paradigmática; Componente 7 Fases; Conceição \& Vasco (2008) 
APÊNDICE B. ANÁLISE DAS NARRATIVAS DOS PACIENTES FOLHA DE COTAÇÃO DE UMA SESSÃO

Sessão:

Avaliador:

Data:

Breve descrição da sessão:

$\underline{\text { Processos narrativos }}$

Observações

Descrição

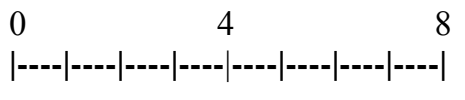

Especificação/concretização

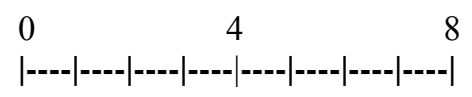

Generalização/abstracção

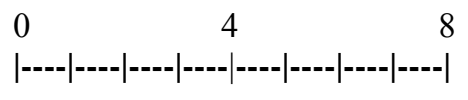

Contextualização

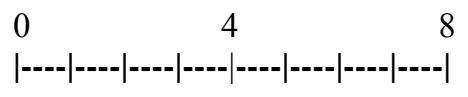

Consciencialização

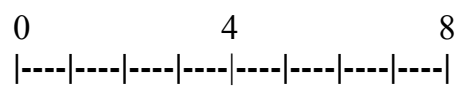

Exploração

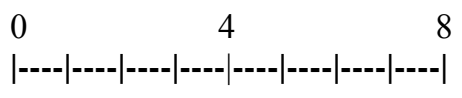

Elaboração

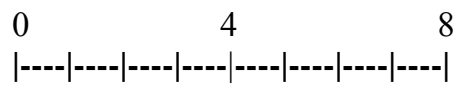

Diferenciação

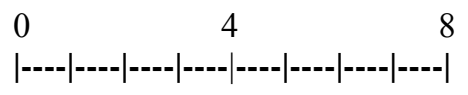


Atribuição de significado

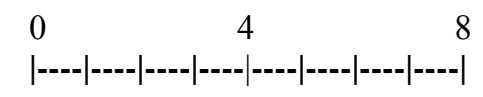

Estabelecimento de relações

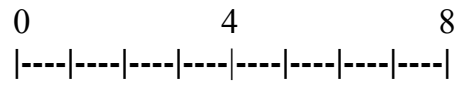

Categorização

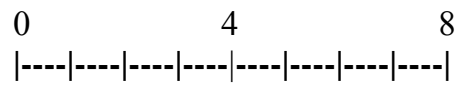

Responsabilização

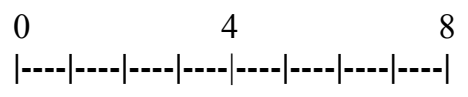

Afirmação

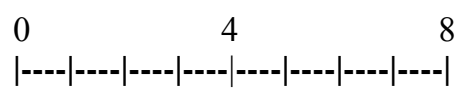

